\title{
Distúrbios gastrointestinais associados à infecção pelo vírus SARS-COV-2: Uma revisão sistemática de literatura
}

\author{
Gastrointestinal disorders associated with SARS-COV-2 virus infection: A systematic Literature \\ review
}

Trastornos gastrointestinales asociados com infeción por el vírus SARS-COV-2: Revisión sistemática de la literatura

\section{Resumo}

Esse trabalho apresenta algumas considerações referente aos distúrbios gastrointestinais em paciente com diagnóstico para o COVID-19, assim como a relação da transmissão fecal-oral. A partir de então, tenciona-se no estudo, gerar ao público leitor, maior esclarecimento sobre a temática da pesquisa. Com isso, objetiva-se analisar artigos de pesquisas e revisões bibliografias voltados aos distúrbios gastrointestinais associados à infecção pelo vírus SARS-COV-2. Para a consolidação de seu objetivo, fez-se necessário um estudo voltado para a revisão sistemática da literatura, o qual utilizou-se 7 artigos publicados no ano de 2020 e 2021, completos e disponíveis para downloads de forma gratuita, no idioma português. Foram excluídos da busca, artigos incompletos, de revisão de literatura, e fora do tempo determinado para a pesquisa. $\mathrm{O}$ estudou mostrou a relação dos sintomas gástricos associado aos pacientes com o vírus SARS-COV-2, assim como apresentou dados sobre meio de cuidados de higiene para o enfretamento da doença. Após a análise dos estudos selecionados conclui-se que, é de grande relevância cientifica a solicitação de radiologias abdominais para o prognóstico do paciente.

Palavras-chave: Complicações; Infecção; Infecção gastrointestinais; SARS-COV-2.

\begin{abstract}
This work presents some considerations regarding gastrointestinal disorders in a patient diagnosed with COVID-19, as well as the relationship between fecal-oral transmission. From then on, the intention of the study is to generate greater clarity on the research topic to the reading public. Thus, the objective is to analyze research articles and bibliographic reviews aimed at gastrointestinal disorders associated with infection by the SARS-VOC-2 virus. For the consolidation of its objective, it was necessary a study focused on the systematic review of the literature, which used 07 articles published in the year 2020 and 2021, complete and available for free downloads, in the Portuguese language. Incomplete articles from the literature review were excluded from the search, and outside the time set for the research. The study showed the relationship of gastric symptoms associated with patients with the SARS-COV-2 virus, as well as presented data on means of hygiene care to cope with the disease. After the analysis of the selected studies, it is concluded that the request for abdominal radiologies for the patient's prognosis is of great scientific relevance.
\end{abstract}

Keywords: Complications; Infection; Gastrointestinal infection; SARS-COV-2. 


\begin{abstract}
Resumen
Este artículo presenta algunas consideraciones sobre los trastornos gastrointestinales en pacientes diagnosticados de COVID-19, así como la relación entre la transmisión fecal-oral. A partir de entonces, el estudio pretende generar más aclaraciones sobre el tema de investigación a los lectores. Así, el objetivo es analizar artículos de investigación y revisiones bibliográficas centradas en los trastornos gastrointestinales asociados a la infección por el virus SARSCOV-2. Para la consolidación de su objetivo fue necesario un estudio dirigido a la revisión sistemática de la literatura, que utilizó 07 artículos publicados en 2020 y 2021, completos y disponibles para descarga gratuita, en portugués. Se excluyeron de la búsqueda los artículos incompletos, los artículos de revisión de la literatura y fuera del tiempo determinado para la búsqueda. El estudio mostró la relación de los síntomas gástricos asociados a los pacientes con el virus SARS-COV-2, así como también presentó datos sobre los medios de cuidado higiénico para enfrentar la enfermedad. Tras analizar los estudios seleccionados, se concluye que es de gran relevancia científica solicitar radiología abdominal para el pronóstico del paciente.
\end{abstract}

Palabras clave: Complicaciones; Infección; Infección gastrointestinal. SARS-COV-2.

\title{
1. Introdução
}

A síndrome respiratória aguda grave 2 (SARS-COV-2), causadora da atual pandemia de COVID-19 que nos acomete, tem tido sua origem suspeitada na China, na província de Wuhan, no Mercado Huana, onde foi possível e acreditado que um animal estivesse envolvido nesse surgimento, de acordo com a similaridade do vírus SARS-COV-2 com o da SARS-COV dos morcegos da região. Acredita-se, atualmente, na possibilidade de esses animais serem hospedeiros para tais agentes infecciosos (Andersen et al., 2020).

A atual pandemia do SARS-COV-2, com as primeiras notificações oficiais em dezembro de 2019, já contaminou, ao redor do mundo, mais de 60 milhões de pessoas, com um positivo número de mais de 38 milhões de recuperados, mas, infelizmente, com a triste quantia de aproximadamente 1,5 milhão de mortes (Zhonghua, 2020).

É uma doença complexa devido ao seu comportamento patológico, que pode evoluir para formas assintomáticas como para formas sintomáticas graves. Comumente, em acometimentos sem complicações mais graves, os principais sintomas são hiposmia ou anosmia, febre, diminuição ou perda do paladar, tosse, congestão nasal, dor de garganta, dispneia, cefaleia, mialgia. Além do acometimento do sistema respiratório, causador de muitas mortes, outros sistemas principais também são gravemente acometidos, como o sistema cardiovascular, o sistema nervoso e o sistema gastrointestinal (Gongora \& Gamez, 2020).

Pesquisas científicas demonstraram recentemente sobre esse envolvimento sistemático gastrointestinal provaram que há uma rota de infecção oral-fecal, pela qual o vírus da SARS-COV-2 invade as células epiteliais das glândulas do estômago, do duodeno, do reto e, em menor extensão, do esôfago (Liny et al., 2020).

Segundo Eder et al, (2020), a proteína que tem sido vista como crucial para a ação infectante deste vírus em questão, até o momento, é a enzima conversora de angiotensina 2, presente principalmente no sistema respiratório, nos epitélios traqueobrônquico e alveolar. Entretanto, essa proteína também é encontrada expressa nas células endoteliais e musculares lisas de vários órgãos viscerais, como no epitélio esofágico, na mucosa gástrica glandular, nos enterócitos, nos colonócitos e nos colangiócitos (Xiao et al., 2020).

Os principais distúrbios gastrointestinais relatados associados a essa infecção são falta de apetite, diarreia, náuseas, vômito, dor abdominal. Em situações de casos que evoluíram com complicações, alguns sintomas mais graves estão presentes, como: fezes com sangue, melena, hematoquezia (Brito et al.,2020).

Com isso, considera-se de grande valia o referido estudo, pois as manifestações gastrointestinais da infecção causada pelo SARS-COV-2 devido à grande prevalência de complicações e evoluções para óbito que essa pandemia vem acarretando.

\section{Metodologia}

A bibliografia utilizada na presente produção científica baseia-se em artigos de pesquisas e de revisão sistemática de 
literatura, procurados com recurso nas bases de dados eletrônicos, nomeadamente Lilacs, PubMed, Scielo, Medline, PsycINFO. Foi também consultada literatura de âmbito acadêmico e Googlescholar.

A questão de pesquisa que norteou a elaboração da presente pesquisa consistiu em: compreender sobre a relação do vírus SARS-COV-2 com os distúrbios gastrointestinais? Estruturado a partir da técnica da revisão sistemática da literatura. Diante desta abordagem Counsell (1997) aponta as seguintes etapas de pesquisa a ser realizada na (RSL) onde (i) é o problema da pesquisa (ii) seleção dos artigos para estudo, (iii) inclusão dos artigos, (iv) obtenção dos dados, (v) analise dos resultados, (vi) discussão dos resultados e (vii) organizar as revisões.

Para Briner et Denyer (2012), para que se possa produzir um estudo voltado para a revisão sistemática, é relevante mencionar que inicialmente deve-se buscar estudos que estejam de acordo com a problemática da pesquisa, bem como analisar os estudos de acordo com sua relevância assim como suas contribuições.

A partir dos resultados encontrados após a busca dos estudos e obedecendo rigorosamente aos critérios de inclusão e exclusão apresentados, será realizada a leitura exaustiva do título e do resumo de cada artigo científico a fim de verificar a sua adequação com a questão norteadora da presente investigação.

A pesquisa de artigos para a presente revisão bibliográfica foi efetuada entre os meses setembro e dezembro de 2020. Foram selecionados os artigos mais recentes, dentre os anos de 2020 e 2021, que foi quando as pesquisas sobre o assunto ficaram mais intensas, pois o conhecimento oficial sobre essa virose da pandemia iniciou no último mês do ano de 2019. Desta forma foi utilizado como critérios de inclusão artigos publicados no ano de 2020 e 2021, completos e disponíveis para downloads de forma gratuita, no idioma português. Foram excluídos da busca, artigos incompletos, de revisão de literatura, e fora do tempo determinado para a pesquisa. 
Figura 1 - Fluxograma do processo de investigação, seleção e inclusão de artigos nas bases da Lillcas, Scielo e Googlescholar.

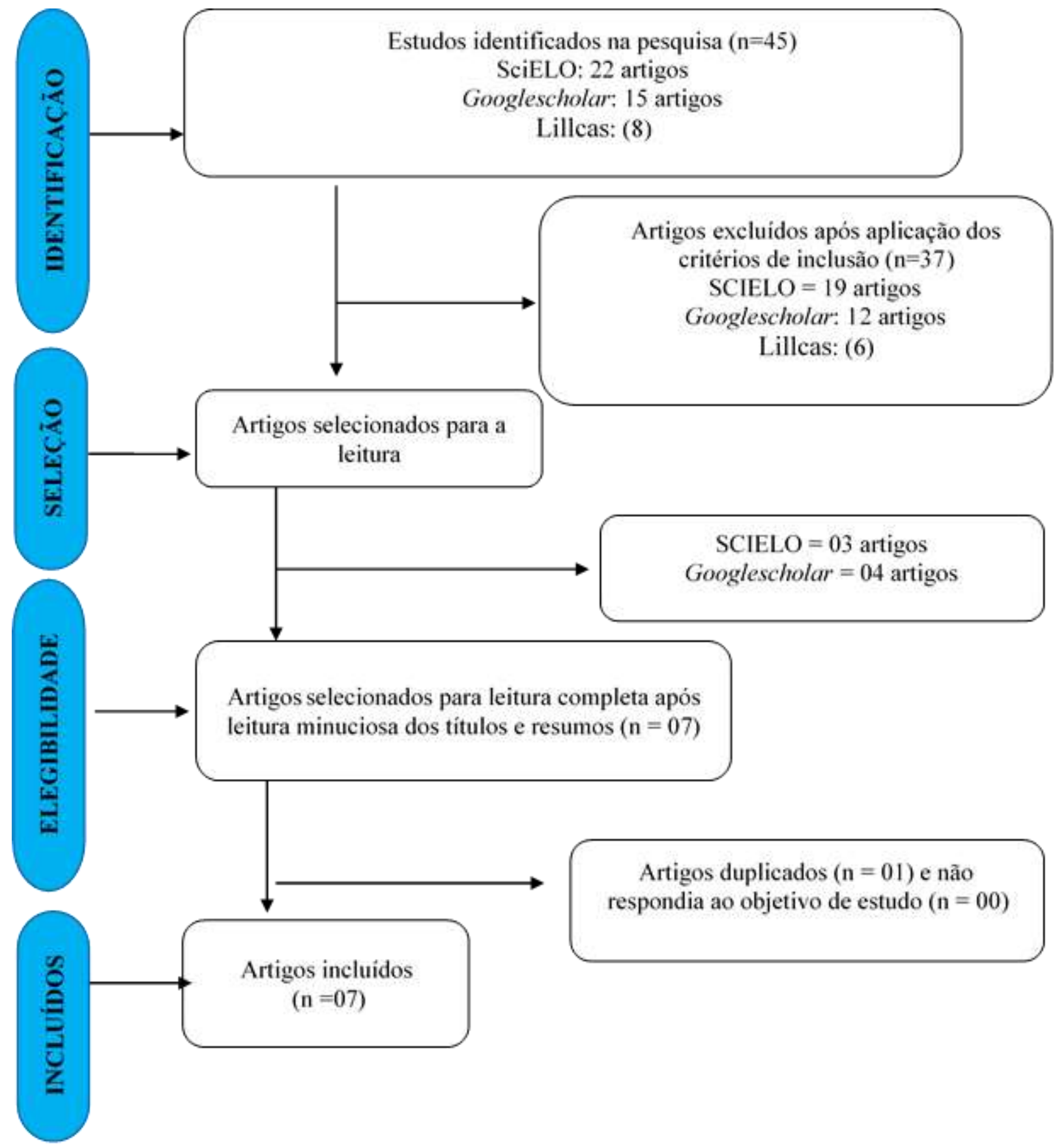

Fonte: Autores (2020).

\section{Resultados}

Para o desenvolvimento deste artigo foram encontrados 45 artigos, os quais estão de acordo com descritores da pesquisa, porém apenas 07 encontram-se de acordo com os critérios de inclusão. Em busca de compreender melhor fez-se necessário a elaboração da Tabela 1, o mesmo segue as descrições dos artigos selecionados, junto as suas respectivas publicações, metodologia e principais resultados. 
Tabela 1. Perfil das produções científicas quanto ao título, ano, metodologia, principais resultados e base de dados.

\begin{tabular}{|c|c|c|c|}
\hline Identificação dos artigos & Metodologia & Principais resultados & Fonte de pesquisa \\
\hline $\begin{array}{l}\text { Sintomas abdominais como } \\
\text { manifestação inicial da } \\
\text { COVID-19: uma série de } \\
\text { casos. (AMARAL } \text { et al., } \\
\text { 2020) } \\
\end{array}$ & $\begin{array}{l}\text { Revisão de } \\
\text { literatura }\end{array}$ & $\begin{array}{l}\text { Mediante estudo realizado, pode-se observar a } \\
\text { presença de distúrbios gastrointestinal em } \\
\text { paciente com Covid-19, a presente afirmação } \\
\text { veio após análise de exames de imagens. }\end{array}$ & SciELO \\
\hline $\begin{array}{l}\text { COVID-19 e o trato } \\
\text { gastrointestinal: o que já } \\
\text { sabemos?. (ALMEIDA e } \\
\text { CHEHTER, 2020). }\end{array}$ & $\begin{array}{l}\text { Revisão de } \\
\text { literatura }\end{array}$ & $\begin{array}{c}\text { Diante do estudo realizado é relevante } \\
\text { mencionar o acometimento gastrointestinal } \\
\text { associado ao vírus SARS-COV-2. }\end{array}$ & SciELO \\
\hline $\begin{array}{l}\text { SARS-COV-2: Uma revisão } \\
\text { para o clínico. (CESPEDES } \\
\text { e SOUZA, 2020). }\end{array}$ & $\begin{array}{l}\text { Revisão de } \\
\text { literatura }\end{array}$ & $\begin{array}{l}\text { Por meio da pesquisa, destaca-se a importância } \\
\text { do pré-diagnóstico da Covid-19, no intuito de } \\
\text { evitar dano maior a saúde e vida do paciente. }\end{array}$ & Googlescholar \\
\hline $\begin{array}{l}\text { Definição de caso Suspeito } \\
\text { da COVID-19: uma revisão } \\
\text { narrativa dos sintomas mais } \\
\text { frequentes entre os casos } \\
\text { confirmados. (ISER, et al., } \\
\text { 2020). }\end{array}$ & $\begin{array}{l}\text { Revisão narrativa } \\
\text { na literatura }\end{array}$ & $\begin{array}{l}\text { Observa-se o quanto a falta do diagnóstico } \\
\text { contribui para o agravamento da doença, desta } \\
\text { forma destaca-se a necessidade de um olhar } \\
\text { mais cientifico para os sintomas apresentado } \\
\text { pelo o paciente, levando em consideração não } \\
\text { somente os sintomas respiratórios, tendo em } \\
\text { vistas que os distúrbios gastrointestinais também } \\
\text { ocorrem em decorrência da Covid-19. }\end{array}$ & SciELO \\
\hline $\begin{array}{l}\text { COVID-19 e o trato } \\
\text { gastrointestinal: } \\
\text { fisiopatologia e evolução } \\
\text { clínica dos pacientes. } \\
\text { (MORAIS, et al., 2020). }\end{array}$ & $\begin{array}{c}\text { Revisão narrativa } \\
\text { da literatura }\end{array}$ & $\begin{array}{c}\text { A pesquisa constatou a presenta do vírus no } \\
\text { TGI, ocasionando danos ao organismo, sendo } \\
\text { que dentre dos principais sintomas pode-se } \\
\text { mencionar a presença de diarreia, perca do } \\
\text { apetite, náusea, vômitos assim como dor } \\
\text { abdominal. }\end{array}$ & Googlescholar \\
\hline $\begin{array}{l}\text { Manifestações } \\
\text { gastrointestinais como } \\
\text { apresentação inicial da } \\
\text { COVID-19 em pediatria } \\
\text { (SOUZA, } \text { et al.,2020 }\end{array}$ & $\begin{array}{l}\text { Revisão da } \\
\text { literatura }\end{array}$ & $\begin{array}{l}\text { Diante do estudo realizado pode-se apresentar } \\
\text { como as principais sintomatologias febre } \\
\text { seguido por sintomas gastrointestinais. O estudo } \\
\text { ainda associou os sintomas gastrointestinais com } \\
\text { o de uma possível crise de apendicite, o qual seu } \\
\text { diagnóstico dar-se mediante a realização de } \\
\text { exame de imagem. }\end{array}$ & Googlescholar \\
\hline $\begin{array}{l}\text { Manifestações sintomáticas } \\
\text { da doença causada por } \\
\text { coronavírus (COVID-19) } \\
\text { em adultos: revisão } \\
\text { sistemática (NETO, et al., } \\
\text { 2021). } \\
\end{array}$ & $\begin{array}{l}\text { Revisão } \\
\text { sistemática }\end{array}$ & $\begin{array}{l}\text { Destaca-se dentre os principais resultados a } \\
\text { presença dos distúrbios gastrointestinais em } \\
\text { paciente com o vírus do SARS-COV-2. }\end{array}$ & Googlescholar \\
\hline
\end{tabular}

Fonte: Autores (2020).

Diante da Tabela 1, percebe-se que as publicações ocorreram de acordo com o recorte temporal, os quais os dados foram publicados. Desta forma destaca-se que a temática vem ganhando crescimento em meio acadêmico. Entretanto os títulos dos artigos abordam sobre a relação da COVID-19 com os distúrbios gastrointestinais. Quanto ao método utilizado nos referidos estudos, pode-se observar que 01 (um) artigo utilizou a revisão sistemática, 03 (três) estudos utilizaram a revisão narrativa da literatura e 03 (três) revisão da literatura. Após o estudo dos artigos, é relevante mencionar que o vírus SARSCOV-2, não está associado apenas às síndromes respiratórias ou as complicações cardiopatas, o mesmo ainda é responsável por distúrbios gastrointestinais.

Os estudos apresentam-se dados semelhantes sobre as sintomatologias da COVID-19 associado aos distúrbios gastrointestinais, os quais são sintomas que merecem maior relevância, tendo como objetivo o prognóstico, assim como dar-se inicial ao tratamento, evitando desta forma o agravamento da doença assim como um possível óbito. 


\section{Discussão}

Segundo os estudos de Cespedes e Souza (2020) o COVID-19 surgiu em Wuhan na China, cientistas evidenciam que o novo coronavírus origina-se em morcegos, sendo que estes animais são facilmente encontrados e consumidos no mercado local. Com o agravamento da doença, bem como a presença do vírus em vários países a OMS - Organização Mundial da Saúde anunciava em 11 de março de 2020 a pandemia da COVID-19. Neste momento as incertezas tomavam de conta do mundo a fora, a medicina era leiga diante da gravidade da doença. Era preciso buscar dentro da ciência evidencias cientificas para salvar vidas.

Inicialmente o vírus apresentava como principais características sintomas gripais, levando o acometimento pulmonar. Os Estudos de Morais et al, (2021) apontam que além da Síndrome Gripal, o indivíduo infectado pelo o vírus também apresentam distúrbios gastrointestinais, os quais é possível encontrar ácido ribonucleico (RNA) do vírus em análise das fezes de pacientes com coronavírus.

Iser et al, (2020), enfatizam que a falta de teste para o diagnóstico de paciente com vírus SARS-COV-2, contribui tanto para a transmissão quanto para a evolução da doença, sendo que muitos pacientes já chegam as unidades de saúde apresentando sinais graves. É relevante mencionar a associação da diarreia prolongada, dor abdominal, vômito, náusea e falta de apetite com o coronavírus. Desta forma Neto et al, (2020) destacam que os distúrbios do trato gastrointestinais (TGI) são sintomas tão evidentes quantos os sintomas respiratórios.

Diante dos estudos realizados, pode-se observar que ambos apresentam as mesmas perspectivas ao que se refere aos distúrbios abdominais em pacientes com sintomatologias voltadas para a COVID-19. Todavia, observou-se ainda que a presença de manifestações gastrointestinais ainda passa por despercebidas. Diante desta abordagem Almeida e Chehter (2020) estima em seus estudos que cerca de 61,3\% dos pacientes com o vírus apresentam sintomas gastrointestinais, os quais apresentam diarreia, dor abdominal, náusea, vômitos e anorexia. Os autores ainda mencionam a presença do vírus em análise das fezes, o que nos leva a compreender a possibilidade de uma possível transmissão fecal-oral.

Corroborado a este mesmo entendimento os estudos de Amaral et al, (2020) destacou a dor abdominal como a mais presente em pacientes que buscam atendimento médico, seguido pela diarreia. Com o avanço das pesquisas voltadas para o SARS-COV-2, os sintomas gastrointestinais tornam-se mais evidentes para o fechamento dos casos. Os quais segundo os autores $61 \%$ dos pacientes apresentam sintomas gastrointestinais no período da doença.

Segundo os estudos de Morais et al, (2021) foi possível observar em paciente com distúrbios gastrointestinais a presença de lesão microvascular do intestino delgado, bem como modificação na flora intestinais, onde a presença destes fatores evolui para a forma mais grave da doença. Contundo, os distúrbios trato gastrointestinal (TGI) pode ser o único sintoma apresentado pelo o paciente com COVID-19, sendo este é dos fatores para o atraso no diagnóstico, o qual favorece a evolução da doença para o estado mais grave.

De acordo com Souza et al, (2020), o distúrbio gastrointestinal decorrente do coronavírus são mais presentes em crianças, tendo em vista, que a dor abdominal é o sintoma mais prevalente no diagnóstico do COVID-19 neste grupo. Desta forma é relevante mencionar a importância de olhar cientifico para todos os sintomas que possam estar associados ao vírus SARS-COV-2.

Verificou-se nesta revisão que mesmo diante dos estudos analisados, ainda é visto a necessidade pesquisas mais detalhadas, bem como uma variedade de abordagem metodológicas, tendo como meta um aparato de informações cientificas ao que se refere às manifestações da COVID-19 causado o distúrbio gastrointestinal. Desta forma pode-se observar que o presente estudo ainda possui uma característica limitada, tendo em vista que o vírus possui como cultura própria o acometimento do sistema respiratório o que torna os estudos voltados para os distúrbios gastrointestinais em pacientes com COVID-19 limitado. 
Receber o diagnóstico positivo para a COVID-19, ainda é algo muito assustador, porém quanto mais cedo se ter este diagnóstico melhor será para o paciente, tendo em vista, que o mesmo terá maior chance de cura, bem como evitar-se o agravamento da doença e um possível óbito.

Em sua trajetória os pacientes com distúrbios gastrointestinais associados ao coronavírus, vivenciam diversos sintomas tendo como os mais presentes, dor abdominal, náusea, vômitos, diarreia, bem como alterações nas enzimas hepáticas. Entretanto podemos concluir mediante este estudo de teóricos o quanto é relevante à realização de teste, bem como solicitação de exames complementares para que se possa fechar o diagnóstico, propondo ao paciente um atendimento eficaz.

Com base neste estudo, pode-se alertar sobre os sintomas gastrointestinais como sintomas presentes em pacientes com COVID-19, bem como a importância de um olhar cientifico direcionado a presença viral do SARS-COV-2 em fezes de indivíduos infectados, o que nos leva a compreender que se pode haver a transmissão fecal-oral. É relevante mencionar como essencial às medidas de higiene como ação no combate da disseminação da doença.

Espera-se com este artigo, suscitar novas pesquisas, sobretudo destacar sobre os distúrbios gastrointestinais presente em pacientes com o COVID-19. Enfim destaca-se como fundamental o uso de radiologias abdominais como ferramenta no prognóstico para o coronavírus.

\section{Conclusão}

Receber o diagnóstico positivo para a COVID-19, ainda é algo muito assustador, porém quanto mais cedo se ter este diagnóstico melhor será para o paciente, tendo em vista, que o mesmo terá maior chance de cura, bem como evitar-se o agravamento da doença e um possível óbito.

Em sua trajetória os pacientes com distúrbios gastrointestinais associados ao coronavírus, vivenciam diversos sintomas tendo como os mais presentes, dor abdominal, náusea, vômitos, diarreia, bem como alterações nas enzimas hepáticas. Entretanto podemos concluir mediante este estudo de teóricos o quanto é relevante à realização de teste, bem como solicitação de exames complementares para que se possa fechar o diagnóstico, propondo ao paciente um atendimento eficaz.

Com base neste estudo, pode-se alertar sobre os sintomas gastrointestinais como sintomas presentes em pacientes com COVID-19, bem como a importância de um olhar cientifico direcionado a presença viral do SARS-COV-2 em fezes de indivíduos infectados, o que nos leva a compreender que se pode haver a transmissão fecal-oral. É relevante mencionar como essencial às medidas de higiene como ação no combate da disseminação da doença.

Espera-se com este artigo, suscitar novas pesquisas, sobretudo destacar sobre os distúrbios gastrointestinais presente em pacientes com o COVID-19. Enfim destaca-se como fundamental o uso de radiologias abdominais como ferramenta no prognóstico para o coronavírus.

\section{Referências}

Almeida, J. F. M. D., \& Chehter, E. Z. (2020). COVID-19 e o trato gastrintestinal: o que já sabemos? Einstein (São Paulo). https://journal.einstein.br/article/covid-19-and-the-gastrointestinal-tract-what-do-we-already-know/.

Amaral, L. T. W., Brito, V. M., Beraldo, G. L., Fonseca, E. K. U. N., Yokoo, P., Talans, A., \& Szarf, G. (2020). Sintomas abdominais como manifestação inicial da COVID-19: uma série de casos. Einstein (São Paulo). https://doi.org/10.31744/einstein_journal/2020rc5831.

Andersen, K. G., Rambaut, A., Lipkin, W. I., Holmes, E. C., \& Garry, R. F. (2020). The proximal origin of SARS-CoV-2. Nature medicine, 26(4), 450-452. https://www.nature.com/articles/s41591-020-0820-9?error=cookies_not_supported\&code=5d04f31a-7879-4af2-96d5-bcdfa2747f4e.

Briner, R. B., \& Denyer, D. (2012). Systematic review and evidence synthesis as a practice and scholarship tool. Handbook of evidence-based management: Companies, classrooms and research, 112-129.

Brito, A. P. S. O., Brito, M. V. H., de jesus Quintino, A., Mansour, I. B., da Silva Récio, C., \& de Azevedo Picanço, N. J. (2020). Manifestações gastrointestinais em pacientes infectados pelo SARS-CoV-2: Revisão sistemática da literatura. Brazilian Journal of Health Review, 3(5), 14570-14583. https://www.brazilianjournals.com/index.php/BJHR/article/view/18317. 
Cespedes, M. S., \& Souza, J. C. (2020). SARS-CoV-2: uma revisão para o clínico.Rev. Assoc. Med. Bras. vol.66 n.4 São Paulo. https://doi.org/10.1590/18069282.66.4.547.

Counsell, C. (1997). Formular perguntas e localizar estudos primários para inclusão em revisões sistemáticas. Annals of internal medicine, 127 (5), $380-387$.

De Morais, L. R., Silva, J. F., Fonseca, L. N., Vieira, M. H. G., de Resende, S. F. R., Simões, S. C., ... \& Rodrigues, V. D. S. S. (2021). COVID-19 e o trato gastrointestinal: fisiopatologia e evolução clínica dos pacientes. Brazilian Journal of Health Review, 4(2), 4556-4569. https://www.brazilianjournals.com/index.php/BJHR/article/view/25659.

Eder, P., Lodyga, M., Dobrowolska, A., Rydzewska, G., \& Kamhieh-Milz, J. (2020). Abordando vários aspectos gastroenterológicos da doença coronavírus 2019. Pol Arch Intern Med , 420-430. https://pubmed.ncbi.nlm.nih.gov/32356641/.

Góngora Gómez, O., \& Gámez Leyva, L. R. (2020). Manifestaciones extrapulmonares de la infección por el nuevo Coronavirus SARS-CoV-2. Revista Habanera de Ciencias Médicas, 19. https://pesquisa.bvsalud.org/controlecancer/resource/pt/biblio-1126910?src=similardocs.

Iser, B. P. M., Sliva, I., Raymundo, V. T., Poleto, M. B., Schuelter-Trevisol, F., \& Bobinski, F. (2020). Definição de caso suspeito da COVID-19: uma revisão narrativa dos sinais e sintomas mais frequentes entre os casos confirmados. Epidemiologia e Serviços de Saúde, 29, e2020233. https://doi.org/10.5123/s167949742020000300018 .

Leite, C., Trindade, E. N., Grillo, L. W., \& Trindade, M. R. M. (2020). Gastrointestinal emergency care during the COVID-19 pandemic: rapid communication. Revista da Associação Médica Brasileira, 66(9), 1187-1189.

Lin, L., Jiang, X., Zhang, Z., Huang, S., Zhang, Z., Fang, Z., \& Shan, H. (2020). Gastrointestinal symptoms of 95 cases with SARS-CoV-2 infection. Gut, 69(6), 997-1001. https://pubmed.ncbi.nlm.nih.gov/32241899/.

Lisboa, C. S., Lima, L. D. O. R., Dayube, M. N., Oliveira, N. S., Almeida, L. M. R., \& Santos, P. S. (2021). Manifestações gastrointestinais em pacientes com Covid-19. Brazilian Journal of Health Review, 4(3), 10620-10638.

Mao, R., Qiu, Y., He, J. S., Tan, J. Y., Li, X. H., Liang, J., \& Chen, M. H. (2020). Manifestations and prognosis of gastrointestinal and liver involvement in patients with COVID-19: a systematic review and meta-analysis. The lancet Gastroenterology \& hepatology, 5(7), 667-678.

Sousa, A. R. D., Carvalho, A. R. B. D., Oliveira, E. M. N. D., Magalhães, R. D. L. B., Moura, M. E. B., \& Freitas, D. R. J. D. (2021). Manifestações sintomáticas da doença causada por coronavírus (COVID-19) em adultos: revisão sistemática. Revista Gaúcha de Enfermagem,42. https://doi.org/10.1590/SciELOPreprints.1436.

Souza, A. L., de Matos, F. A., Flintz, R. A., Marliere, R. C. C., Presti, M. B. L., \& Lopes, C. (2020). Manifestações gastrointestinais como apresentação inicial da COVID-19 em pediatria. Residência Pediátrica; 2020: Ahead of Print. https://doi.org/10.25060/residpediatr.

Surveillances, V. (2020). The epidemiological characteristics of an outbreak of 2019 novel coronavirus diseases (COVID-19) China, 2020. China CDC weekly, 2(8), 113-122. https://pubmed.ncbi.nlm.nih.gov/32064853/.

Xiao, F., Tang, M., Zheng, X., Liu, Y., Li, X., \& Shan, H. (2020). Evidence for gastrointestinal infection of SARS-CoV-2. Gastroenterology, 158(6), 18311833. https://www.ncbi.nlm.nih.gov/pmc/articles/PMC7130181/.

Zhou, Z., Zhao, N., Shu, Y., Han, S., Chen, B., \& Shu, X. (2020). Effect of gastrointestinal symptoms in patients with COVID-19. Gastroenterology, 158(8), 2294. 